\title{
EGFR tyrosine kinase inhibitors (TKIs) for adjuvant therapy of early-stage non-small cell lung cancer (NSCLC): ready for the clinic?
}

\author{
Conor E. Steuer, Suresh S. Ramalingam \\ Department of Hematology \& Medical Oncology, Winship Cancer Institute of Emory University, Atlanta, GA, USA \\ Correspondence to: Suresh S. Ramalingam, MD. 1365 C Clifton Rd NE, Atlanta, Georgia 30322, USA. Email: ssramal@emory.edu. \\ Comment on: Liang W, Cai K, Chen C, et al. Society for Translational Medicine consensus on postoperative management of EGFR-mutant lung \\ cancer (2019 edition). Transl Lung Cancer Res 2019;8:1163-73.
}

Submitted Apr 10, 2020. Accepted for publication Apr 23, 2020.

doi: $10.21037 /$ tlcr-2020-13

View this article at: http://dx.doi.org/10.21037/tlcr-2020-13

For patients diagnosed with early-stage non-small cell lung cancer (NSCLC), there is a need for improvement as present cure rates are relatively modest; the 5 -year survival rates range from approximately $75 \%$ for stage I disease to $30 \%$ for stage 3 disease with surgical resection alone (1). The addition of adjuvant platinum-based chemotherapy results in $4-15 \%$ absolute improvement in the 5 -year survival rate; hence it has been adopted as the standard of care for stage IB-III NSCLC (2). However, over the past 15 years, it has become clear that NSCLC is not a monolithic disease. Molecular profiling techniques have identified biologically distinct subsets of NSCLC, with EGFR-mutant (EGFRm) NSCLC being one of the most investigated and defined. For patients with metastatic EGFRm NSCLC, multiple randomized studies have confirmed the superiority of EGFR tyrosine kinase inhibitor (TKI) to chemotherapy in terms of efficacy, quality of life (QOL), and toxicity (3). Based on these therapeutic gains, the next logical step of studying EGFR inhibitors for patients with early-stage NSCLC bearing EGFR mutations has been undertaken by several groups. At the outset, it is important to note that the overall prognosis for EGFRm NSCLC patients with early-stage disease appears more favorable compared to patients with wild-type EGFR [hazard ratio (HR) 0.40-0.51] (4,5). It is also relevant to mention here that EGFR TKI therapy has not resulted in cure for patients with metastatic disease; acquired resistance invariably develops regardless of the extent and duration of clinical benefit. As the first set of studies report on the outcomes for early-stage NSCLC patients treated with
EGFR TKI, it is timely to discuss the implications of the early observations to clinical practice.

The accompanying article by Liang et al. proposes consensus guidelines for the post-operative management of EGFRm NSCLC and seeks to address many of the questions around the care for resected EGFRm NSCLC. The consensus panel studied the levels of evidence to support practice guidelines for pertinent clinical questions on this topic. The paper also includes opinions from a wide range of experts from Asia and Europe on the consensus guidelines. Based on the data available, Liang et al. gave the use of adjuvant TKI for EGFRm NSCLC a "strong" recommendation (6). We believe that a discussion on this topic is timely and necessary. It is our hope that rapid adoption of CT screening for early detection of lung cancer will result in a higher proportion of patients being diagnosed with early-stage disease. Therefore, answers to important questions regarding management of oncogeneaddicted lung cancer patients with early-stage NSCLC are urgently needed.

The BR-19 trial was among the earliest to evaluate the role of EGFR TKIs in resected NSCLC (gefitinib vs. observation); however, this was carried out in an unselected patient population with only 15 patients with an EGFR mutation. The study was closed early for futility with an overall survival HR of 1.24; even in the subgroup analysis for patients with EGFRm, there was no difference in overall survival (7). A single arm phase 2 study (SELECT) examined erlotinib for resected EGFRm NSCLC with the primary endpoint of 2-year disease-free survival 
(DFS). For the cohort of 100 patients, the 2-year DFS was promising at $88 \%$ (8). Similarly, the EVAN trial conducted in China compared adjuvant erlotinib to platinumbased chemotherapy. Approximately 100 patients were enrolled and the primary endpoint was met with a 2 -year DFS of $81.4 \%$ in the erlotinib group and $44.6 \%$ in the chemotherapy group [relative risk (RR) 1.8] (9). Finally, a retrospective, single institution study showed a favorable DFS for patients that received adjuvant TKI $v s$. those who did not (HR 0.43) (4). These early observations extended support to the safety of adjuvant EGFR TKIs, but were not confirmatory of clinical benefit.

The ADJUVANT and the RADIANT phase 3 trials are cited frequently to make the case for or against adjuvant TKI therapy. The RADIANT study compared erlotinib to placebo in a general population of resected NSCLC; efficacy in EGFRm patients was a secondary endpoint. The study failed to demonstrate an improvement in DFS for erlotinib therapy (HR 0.90). For the 161 patients with EGFR mutations, the median DFS was higher for the erlotinib group (46.4 vs. 28.5 months, HR 0.61). However, due to the hierarchical statistical analysis, the difference in DFS was not statistically significant (10). The ADJUVANT study, conducted in China, randomized stage II/IIIA EGFRm NSCLC patients to either gefitinib or cisplatin plus vinorelbine adjuvant therapy. The median DFS was superior for gefitinib vs. chemotherapy (28.7 vs. 18.0 months, HR 0.60) (11). The overall survival results from these two trials have not been reported.

Despite the promising results observed in these two randomized trials, we contend that the data do not support the adoption of adjuvant TKI therapy for routine clinical use. The primary reason for our conclusion is the lack of survival data with adjuvant therapy. Though DFS has been validated as a surrogate marker of overall survival in the adjuvant setting for certain diseases such as colon and breast cancer, there is no basis for this assumption in NSCLC $(12,13)$. Recent evidence in NSCLC that DFS may serve as an appropriate surrogate for chemotherapy in NSCLC does not imply that the same can be said for targeted therapies (14). Furthermore, there is no evidence that 2 years of adjuvant therapy results in cure and longterm survival for EGFRm patients, given the limited follow up from the published studies. It is possible that adjuvant therapy merely delays metastatic disease, without altering the natural history of the disease. In the ADJUVANT study as an example, it is not apparent that positron emission tomography (PET) scans and brain magnetic resonance imaging (MRI) were required for study entry. Given that $65 \%$ of patients enrolled had stage 3 disease, it is possible that there were metastatic patients unintentionally included in the analysis. Furthermore, there appears to be a converging of the DFS curves quickly at the two-year mark, leading to the thought that the adjuvant TKI delayed disease recurrence, without eradicating the micrometastatic disease.

Another important issue relates to the fact that all subsets of EGFRm NSCLC cannot be considered as one entity; there is ample evidence in the metastatic setting that EGFR exon 19 deletions confer a higher degree of sensitivity to EGFR TKIs than exon 21 L858R mutations, thus raising the possibility that these differences may impact outcomes in the adjuvant setting (15). Furthermore, the more uncommon EGFR mutations, such as in exon 18, were excluded from the ADJUVANT study. These mutations have been shown to be sensitive to EGFR TKIs afatinib and osimertinib in the metastatic setting, and how these mutations will fit into the EGFRm NSCLC adjuvant algorithm remains to be seen $(16,17)$.

In the majority of trials discussed here, the use of TKI typically is studied after patients have received adjuvant chemotherapy. In the EVAN and ADJUVANT studies, EGFR TKI was used in the place of chemotherapy. Given the proven increased survival benefit provided by adjuvant chemotherapy, we do not recommend the exclusion of chemotherapy for EGFRm NSCLC. The IPASS study documented the higher clinical efficacy of systemic chemotherapy for EGFRm patients compared to wild type NSCLC; extrapolating from this observation, it is conceivable that adjuvant chemotherapy may have an even greater degree of benefit for EGFRm patients. Another factor complicating the issue is the promising recent observations in metastatic NSCLC with the addition of chemotherapy to EGFR inhibition (18). It raises the interesting possibility that chemotherapy in combination with EGFR TKI might be the most effective strategy moving forward. The duration of EGFR TKI for earlystage disease is another unsettled issue. The 2-year time period utilized in various trials might be insufficient; the duration of adjuvant imatinib for gastrointestinal stromal tumors (GISTs) is at least 3 years (19). We should also consider the duration of therapy on tolerance and QOL. For example, in the RADIANT study the median duration of erlotinib was only 11.9 months and $44.4 \%$ of patient required a dose reduction (10).

For all these reasons, we do not recommend the routine 
use of adjuvant EGFR TKI for patients with resected NSCLC; ongoing phase 3 trials, such as the ALCHEMIST (CT02193282; erlotinib vs. observation) and the ADAURA (NCT02511106; osimertinib vs. placebo) will provide definitive evidence to inform clinical practice. Recently, it was announced that the ADAURA study will be unblinded early due to "overwhelming efficacy" in favor of osimertinib in the primary endpoint, DFS. We eagerly await the data to become available as well as the OS results. We are also excited about technological advances such as with the use of circulating tumor cells and cell-free DNA evaluation that might allow for identification of minimal residual disease in patients with resected NSCLC, thus leading to truly personalized adjuvant therapy for early-stage NSCLC.

\section{Acknowledgments}

Funding: None.

\section{Footnote}

Provenance and Peer Review: This article was commissioned by the editorial office, Translational Lung Cancer Research. The article did not undergo external peer review.

Conflicts of Interest: Both authors have completed the ICMJE uniform disclosure form (available at http://dx.doi. org/10.21037/tlcr-2020-13). SSR serves as an unpaid editorial board member of Translational Lung Cancer Research. CES reports other from Astrazeneca, other from Eli Lilly, other from Bergen Bio, other from Armo, other from AbbVie, outside the submitted work; SSR reports grants and other from Amgen, other from Abbvie, grants and other from Astra Zeneca, grants and other from BMS, other from Genentech, other from Roche, grants and other from Merck, grants and other from Takeda, grants from Tesaro, grants from Advaxis, outside the submitted work. The authors have no other conflicts of interest to declare.

Ethical Statement: The authors are accountable for all aspects of the work in ensuring that questions related to the accuracy or integrity of any part of the work are appropriately investigated and resolved.

Open Access Statement: This is an Open Access article distributed in accordance with the Creative Commons Attribution-NonCommercial-NoDerivs 4.0 International License (CC BY-NC-ND 4.0), which permits the non- commercial replication and distribution of the article with the strict proviso that no changes or edits are made and the original work is properly cited (including links to both the formal publication through the relevant DOI and the license). See: https://creativecommons.org/licenses/by-nc-nd/4.0/.

\section{References}

1. Lang-Lazdunski L. Surgery for nonsmall cell lung cancer. Eur Respir Rev 2013;22:382-404.

2. Pignon JP, Tribodet H, Scagliotti GV, et al. Lung adjuvant cisplatin evaluation: a pooled analysis by the LACE Collaborative Group. J Clin Oncol 2008;26:3552-9.

3. Lee CK, Wu YL, Ding PN, et al. Impact of Specific Epidermal Growth Factor Receptor (EGFR) Mutations and Clinical Characteristics on Outcomes After Treatment With EGFR Tyrosine Kinase Inhibitors Versus Chemotherapy in EGFR-Mutant Lung Cancer: A MetaAnalysis. J Clin Oncol 2015;33:1958-65.

4. D'Angelo SP, Janjigian YY, Ahye N, et al. Distinct clinical course of EGFR-mutant resected lung cancers: results of testing of 1118 surgical specimens and effects of adjuvant gefitinib and erlotinib. J Thorac Oncol 2012;7:1815-22.

5. Marks JL, Broderick S, Zhou Q, et al. Prognostic and therapeutic implications of EGFR and KRAS mutations in resected lung adenocarcinoma. J Thorac Oncol 2008;3:111-6.

6. Liang W, Cai K, Chen C, et al. Society for Translational Medicine consensus on postoperative management of EGFR-mutant lung cancer (2019 edition). Transl Lung Cancer Res 2019;8:1163-73.

7. Goss GD, O'Callaghan C, Lorimer I, et al. Gefitinib versus placebo in completely resected non-small-cell lung cancer: results of the NCIC CTG BR19 study. J Clin Oncol 2013;31:3320-6.

8. Pennell NA, Neal JW, Chaft JE, et al. SELECT: A Phase II Trial of Adjuvant Erlotinib in Patients With Resected Epidermal Growth Factor Receptor-Mutant Non-SmallCell Lung Cancer. J Clin Oncol 2019;37:97-104.

9. Yue $\mathrm{D}, \mathrm{Xu} \mathrm{S}$, Wang Q, et al. Erlotinib versus vinorelbine plus cisplatin as adjuvant therapy in Chinese patients with stage IIIA EGFR mutation-positive non-small-cell lung cancer (EVAN): a randomised, open-label, phase 2 trial. Lancet Respir Med 2018;6:863-73.

10. Kelly K, Altorki NK, Eberhardt WE, et al. Adjuvant Erlotinib Versus Placebo in Patients With Stage IBIIIA Non-Small-Cell Lung Cancer (RADIANT): A Randomized, Double-Blind, Phase III Trial. J Clin Oncol 
2015;33:4007-14.

11. Zhong WZ, Wang Q, Mao WM, et al. Gefitinib versus vinorelbine plus cisplatin as adjuvant treatment for stage II-IIIA (N1-N2) EGFR-mutant NSCLC (ADJUVANT/ CTONG1104): a randomised, open-label, phase 3 study. Lancet Oncol 2018;19:139-48.

12. Saad ED, Squifflet P, Burzykowski T, et al. Disease-free survival as a surrogate for overall survival in patients with HER2-positive, early breast cancer in trials of adjuvant trastuzumab for up to 1 year: a systematic review and meta-analysis. Lancet Oncol 2019;20:361-70.

13. Martin M, Holmes FA, Ejlertsen B, et al. Neratinib after trastuzumab-based adjuvant therapy in HER2-positive breast cancer (ExteNET): 5-year analysis of a randomised, double-blind, placebo-controlled, phase 3 trial. Lancet Oncol 2017;18:1688-700.

14. Mauguen A, Pignon JP, Burdett S, et al. Surrogate endpoints for overall survival in chemotherapy and radiotherapy trials in operable and locally advanced lung cancer: a re-analysis of meta-analyses of individual patients' data. Lancet Oncol 2013;14:619-26.

15. Wang H, Huang J, Yu X, et al. Different efficacy of EGFR tyrosine kinase inhibitors and prognosis in patients with

Cite this article as: Steuer CE, Ramalingam SS. EGFR tyrosine kinase inhibitors (TKIs) for adjuvant therapy of earlystage non-small cell lung cancer (NSCLC): ready for the clinic? Transl Lung Cancer Res 2020;9(5):1720-1723. doi: 10.21037/ tlcr-2020-13 subtypes of EGFR-mutated advanced non-small cell lung cancer: a meta-analysis. J Cancer Res Clin Oncol 2014;140:1901-9. Erratum in: J Cancer Res Clin Oncol. 2014 Nov; 140(11):1911.

16. Yang JC, Sequist LV, Geater SL, et al. Clinical activity of afatinib in patients with advanced non-small-cell lung cancer harbouring uncommon EGFR mutations: a combined post-hoc analysis of LUX-Lung 2, LUX-Lung 3 , and LUX-Lung 6. Lancet Oncol 2015;16:830-8.

17. Cho JH, Lim SH, An HJ, et al. Osimertinib for Patients With Non-Small-Cell Lung Cancer Harboring Uncommon EGFR Mutations: A Multicenter, OpenLabel, Phase II Trial (KCSG-LU15-09). J Clin Oncol 2020;38:488-95.

18. Noronha V, Patil VM, Joshi A, et al. Gefitinib Versus Gefitinib Plus Pemetrexed and Carboplatin Chemotherapy in EGFR-Mutated Lung Cancer. J Clin Oncol 2020;38:124-36.

19. Laurent M, Brahmi M, Dufresne A, et al. Adjuvant therapy with imatinib in gastrointestinal stromal tumors (GISTs)review and perspectives. Transl Gastroenterol Hepatol 2019;4:24. 